\title{
Periocular Biometrics for Iris Recognition: A Review
}

\author{
Nirgish Kumar ${ }^{1}$, Komal $^{2}$ \\ ${ }^{1}$ Research Scholar, Faculty of Engineering, Rama University, Kanpur, India \\ ${ }^{2}$ AP, Faculty of Engineering, Rama University, Kanpur, India
}

\begin{abstract}
Periocular refers to the facial region in the vicinity of the eye, including eyelids, lashes and eyebrows. While face and irises have been extensively studied, the periocular region has emerged as a promising trait for unconstrained biometrics, following demands for increased robustness of face or iris systems. With surprisingly high discrimination ability, this region can be easily obtained with existing setups for face and iris, and the requirement of user cooperation can be relaxed, thus facilitating the interaction with biometric systems. It is also available over a wide range of distances even when the iris texture cannot be reliably obtained (low resolution) or under partial face occlusion (close distances). Here, we review the state of the art in periocular biometrics research. A number of aspects are described, including: i) existing databases, ii) algorithms for periocular detection and/or segmentation, iii) features employed for recognition, iv) identification of the most discriminative regions of the periocular area, v) comparison with iris and face modalities, and vi) soft-biometrics (gender/ethnicity classification),. This work is expected to provide an insight of the most relevant issues in periocular biometrics, giving a comprehensive coverage of the existing literature and current state of the art. Moreover, we will use different feature extraction techniques such as LBP, PCA, and ICA for pre-processing of periocular biometrics. Comparative Analysis with other competent technologies is also the essential part of this research work.
\end{abstract}

Keywords: Acquisition, Biometric System, Periocular, iris recognition, iris, databases etc.

\section{Introduction}

Aims:

Identification and authentication of any human will be becoming very important in present time. In the surrounding where electronics devices will more commonly used and there is a need for accurate and secured authentication. Old techniques such as passwords, ID cards, are not accurate and secure. Thus, there shall be increasing to implements for automatic authentication process.

\section{Biometric System:}

The word "biometrics" comes from the Greek words "bio" and "metric," meaning "life computation" The unparalleled of a personally, physiological and behavioral, characteristics is the basis for the science of biometrics. Typical physiological features measured include an individual's fingerprints, face, retina, iris, DNA and hand geometry.

\section{Periocular:}

The term "periocular" comes from the prefix, "peri-", meaning "around or about", and the genesis word, "ocular", meaning "of a relating to the eye". In biometrics, the term has been applied to both a small region including the eye, eyelids, and lashes.

\section{Acquisition:}

Researchers have explored multiple kinds of acquisition techniques in iris recognition. The facial region in the immediate vicinity of the eye. Acquisition of the periocular biometric is anticipated to require less subject cooperation while permitting a larger depth of field compared to traditional ocular biometric. Traits (retina, iris, conjunctiva, periocular.)

\section{Objective}

In this research work following of aspect will be investigated and implemented:

- The effectiveness of incorporating the eyebrows,

- An use of side information (left or right) in matching,

- The manual versus automatic segmentation schemes,

- The local versus global feature extraction schemes,

- The fluidization of face and periocular biometrics,

- An use of the periocular biometric in partially occluded face image

- An effect of pose variation and occlusion,

- An effect of masking the iris and eye region,

- Development of novel technique for periocular recognition,

- Comparative Analysis.

Periocular Biometric systems are applied for the unique identification of an individual by evaluating one or more distinguishing biological traits. Authentication plays a major role as a first line of defense against intruders. The number of systems that have been compromised is ever increasing and biometric verification is any means by which a person can be uniquely identified by evaluating one or more distinguishing biological traits. Periocular biometric recognition is based on the appearance of the region around the eye. The performance of iris recognition is affected if iris is captured at a distance, also affected for subjects who are blind or have cataracts and the performance of face recognition is affected by lighting changes, hair of the person, the age and if the person wear glasses. Periocular recognition [1] is useful in applications where it is difficult to implement the iris and the face biometrics. Acquisition of the Periocular biometrics has require to large number of user cooperation. In the existing work, After capturing the 


\section{International Journal of Science and Research (IJSR) \\ ISSN (Online): 2319-7064}

Index Copernicus Value (2015): 78.96 | Impact Factor (2015): 6.391

periocular region, feature extraction method is done using Local binary pattern (LBP), Histograms of Oriented Gradients (HOGs) and Scale Invariant Feature Transform (SIFT). The human and the machine performance are analyzed based on these algorithms and this not a complete automated system. In this manual intervention is required for the recognition of the features that is obtained using those algorithms.
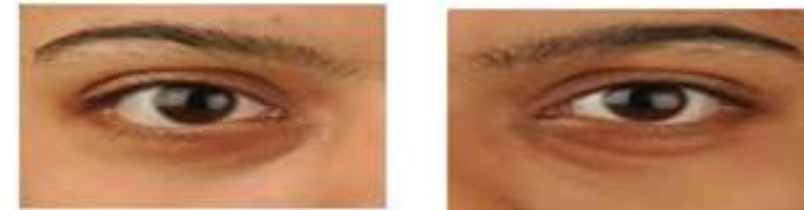

Figure 1: Original Left and Right Periocular Images

(A) J.R.Lyle et al (2010) proposed soft biometric classification using periocular region features in [5]. The focus is on gender and ethnicity classification of individuals using periocular images. The core is to focus whether periocular images carry enough information to reliably obtain similar soft biometric information to that obtained from face images. This paper describes a soft biometric classification approach using appearance based periocular features. The soft biometric information thus obtained can be effectively used for improving the performance of existing periocular based recognition approaches.

(B) D.L.Woodard et al (2010) described periocular region appearance cues for biometric identification in [6]. The lowlevel features extracted from the periocular region can be effectively used for identification. The chief novelty in this work lies in our use of only the level-two periocular features based on skin texture and color information to perform identification corresponding author only.

To this effect, mask the eye in the periocular region thus removing the iris and various level-one features. Although removal of the eye from a periocular region image may seem like a heavy loss of discriminating information, it could be potentially advantageous as the level-one features are highly sensitive to the opening and closing of the eyes and may end up influencing the texture features adversely).

\section{Expected Impact on Academics/Industry}

In spite of the tremendous progress made in ocular biometrics, there are significant provocation encountered by these systems:

(1) The iris is a moving object with a small surface area that is located within the independently transfer eyeball. The eyeball itself is located within another moving object-the head. Therefore, convey localizing the iris in eye images obtained at a distance in unconstrained environments can be difficult [11]. Furthermore, since the iris is generally imaged in the near-infrared (NIR) portion (700-900 nm) of the electromagnetic (EM) spectrum, proper invisible lighting is required to illuminate it prior to image accession.
(2) The size of an iris is very small contrast to that of a face. Face images get with low decision sensors or large standoff distances offer very little or no data; about iris texture.

(3) Even under ideal shape characterized by appreciative lighting conditions and an optimal deadlock distance, if the subject blinks or closes his eye, the iris data cannot be reliably get.

(4) Retinal vasculature cannot be effortless, imaged unless the subject is collective. In addition, the imaging device has to be in close presence to the eye.

(5) While conjunctiva vasculature can be imaged at a distance, the curvature of the sclera, the secular likeness. in the image, and the fineness of the vascular patterns can surprise the feature extraction and similar modules of the biometric system [12].

The performance of periocular recognition could be further enhanced by incorporating the information related to the eye shape. The applications of biometrics will be divided into the following three main bodies.

Commercial: applications such as computer network sign in, electronic data security, E-commerce, Network access, ATM, credit card, bodily access control, cellular phone, PDA, medical records database, and distance learning.

Government: applications such as national ID card, correctional, solution, driver's license, social security, welfare disbursement, border control, and passport control.

Forensic: applications such as corpse distinguish, criminal investigation, terrorist identification, parenthood resolution, and missing children.

\section{Major Inputs (Infrastructure) Requirement}

- Face data set- FDS is a database of static images of human faces.

- Region Extraction- The extraction of regions from multiple images simultaneously with their correspondences.

- Local binary patterns- A type of visual descriptor used for classification in computer vision, and particular case of the Texture Spectrum model.

- Periocular recognition- Periocular recognition has gained attention recently due to demands of increased robustness of face or periocular in less controlled of scenarios.

- Scale-invariant feature transform- An algorithm is computer vision to detect and describe to local features in images.

- Academic Service: Computer Science \& engineering, Department Faculty of Engineering \& Technology, Rama University, Rama Kanpur, Rama City, G.T. Road, Mandhana, Kanpur (U .P.) $208 \quad 017$ Software Requirement: Matlab-R2015a 


\section{International Journal of Science and Research (IJSR) \\ ISSN (Online): 2319-7064}

Index Copernicus Value (2015): 78.96 | Impact Factor (2015): 6.391
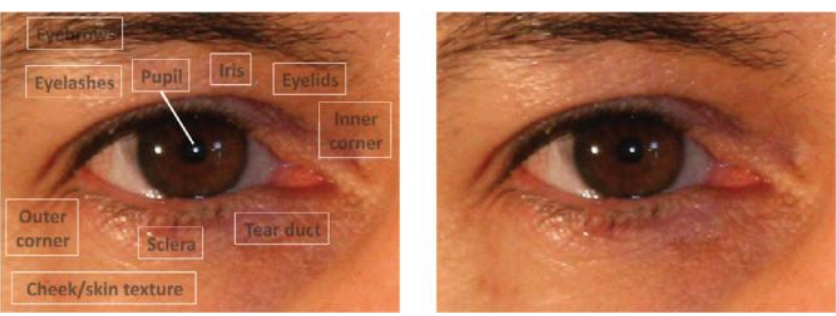

\section{Global versus Local Matcher}

Most image matching schemes can be categorized as being global or local based on whether the features are extracted from the entire image (or a region of interest) or from a set of local regions. Representative global image features include those based on color, shape, and texture [18]. Global features are typically represented as a fixed length vector, and the matching process simply compares these fixed length vectors, which is very time efficient. On the other hand, a local feature-based approach first detects a set of key points and encodes each of the key points using the surrounding pixel values, resulting in a local key point descriptor [19], [20]. Then, the number of matching key points between two images is used as the match score. Since the number of key points varies depending on the input image, two sets of key points from two different images.

\section{References}

[1] H. Han and A. K. Jain. Age, gender and race estimation from nconstrained face images. MSU Technical Report MSU-CSE-14-5, Michigan State University, 2014.

[2] U. Park, A. Ross, and A. Jain. Periocular biometrics in the visible spectrum: A feasibility study. In IEEE 3rd International Conference on Biometrics: Theory, Applications, and Systems, 2009. BTAS '09., pages 16, Sept. 2009

[3] NIST. Face recognition grand challenge database. http://www.frvt.org/FRGC/.

[4] J. Lyle, P. Miller, S. Pundlik, and D. Woodard. Soft biometric classification using periocular region features. In Fourth IEEE International (BTAS), 2010, pages 1-7, Sept. 2010

[5] D. Woodard, S. Pundlik, P. Miller, R. Jillela, and A. Ross.On the fusion of periocular and iris biometrics in non-ideal imagery. (ICPR), 2010, pages 201-204, Aug. 2010.

[6] J.Philips.Multiple biometrics grand challenge.http://face.nist.gov/mbgc/.

[7] L. Simpson, G. Dozier, J. Adams, D. Woodard, P. Miller, K. Bryant, and G. Glenn. Genetic amp; evolutionary type ii feature extraction for periocularbased biometric recognition. In IEEE Congress on Evolutionary Computation (CEC), 2010, pages 1-4, July 2010.

[8] S. Bharadwaj, H. Bhatt, M. Vatsa, and R. Singh. Periocular biometrics: When iris recognition fails. In Fourth IEEE 2010, pages 1-6, Sept. 2010.

[9] H. Proenca, S. Filipe, R. Santos, J. Oliveira, and L. Alexandre. The ubiris.v2: A database of visible wavelength iris images captured on-the-move and at-adistance. IEEE Transactions on Pattern Analysis and Machine telligence, 32(8):1529-1535, Aug. 2010.
[10]P. Miller, J. Lyle, S. Pundlik, and D. Woodard. Performance evaluation of local appearance based periocular recognition.

[11] J. Matey, D. Ackerman, J. Bergen, and M. Tinker, "Iris recognition in less constrained environments,", pp. 107131,2008

[12]S. Crihalmeanu, A. Ross, and R. Derakhshani, "Enhancement and registration schemes for matching conjunctival vasculature," in Proc. Int. Conf. Biometrics (ICB), 2009, pp. 1240-1249.

[13] U. Park, R. Jillela, A. Ross, and A. Jain. Periocular biometrics in the visible spectrum. IEEE Transactions on information Forensics and Security,, 6(1):96-106, March 2011.

[14] Ramli Adnan, Fazlina Ahmat Ruslan, Abd Manan Samad \& Zainazlan Md Zain, "New Artificial Neural Network and Extended Kalman Filter Hybrid Model prediction of flood water level", 2013

[15] J. Bekios-Calfa, J. M. Buenaposada, and L. Baumela. Robust gender recognition by exploiting facial attributes dependencies. Pattern Recogn. Lett., 36:228-234, January 2014.

[16] P. Dago-Casas, D. Gonzalez-Jim ' enez, ' L. Long-Yu, and Jose' Luis Alba Castro. Single- and cross- database benchmarks for gender classification under unconstrained settings. In ICCV'11 -Be FIT 2011, November 2011.

[17] Laurent El Shafey, Elie Khoury, and Sebastien 'Marcel. Audio-visual gender recognition in uncontrolled environment using variability modeling techniques. 2014.

[18] A. Smeulders, M.Worring, S. Santini, A. Gupta, and R. Jain, "Contentbased image retrieval at the end of the early years," IEEE Trans. Pattern Anal. Mach. Intell., vol. 22, no. 12, pp. 1349-1380, Dec. 2000.

[19] C. Schmid and R. Mohr, "Local grayvalue invariants for image retrieval," IEEE Trans. Pattern Anal. Mach. Intell., vol. 19, no. 5, pp. 530-535, May 1997.

[20]K. Mikolajczyk and C. Schmid, "A performance evaluation of local descriptors," IEEE Trans. Pattern Anal. Mach. Intell., vol. 27, no. 10, pp. 1615-1630, Oct. 2005.

[21] S. A. Khan, M. Ahmad, M. Nazir, and N. Riaz. A comparative analysis of gender classification techniques. International Journal of Bio-Science and BioTechnology, 5(4):223-244, 2013.

[22] Haoyu Ren and Ze-Nian Li. Gender recognition using complexity-aware local features. In Pattern Recognition (ICPR), 2014 2389-2394, Aug 2014.

[23] Caifeng Shan. Learning local binary patterns for gender classification on real-world face images. Pattern Recognition Letters, 33(4):431 - 437, 2012. Intelligent Multimedia Interactivity.

[24] J.E. Tapia and C.A. Perez. Gender classification based on fusion of different spatial scale features selected by mutual information from histogram of $\mathrm{lbp}$, intensity, and shape. Information Forensics and Security, IEEE Transactions on, 8(3):488-499, March 2013.

[25]A. Murugan G. Savithiri, "Fragmented Iris Recognition System using BPNN", International Journal of Computer Applications, Vol 36- No.4, pp.28-33, 2011.

\section{Volume 6 Issue 7, July 2017 www.ijsr.net}




\section{International Journal of Science and Research (IJSR) \\ ISSN (Online): 2319-7064}

Index Copernicus Value (2015): 78.96 | Impact Factor (2015): 6.391

[26] Omaima N. Ahmad AL-Allaf, Abdelfatah Aref Tamimi, Shahlla A. AbdAlKader, "Artificial Neural Networks for Iris Recognition System: Comparisons between Different Models, Architectures and Algorithms", International Journal of Information and Communication Technology Research, Vol. 2,No. 11, pp. 795-803, 2012

[27] Vivek Srivastava, Bipin Kumar Tripathi,Vinay Kumar Pathak, "Biometric Recognition By Hybridization Of Evolutionary Fuzzy Clustering With Functional Neural Networks", Springer, pp. 13, 2012.

[28] Hamed Ghodrati, Mohammad Javad Dehghani, Habibolah Danyali,, "Iris Feature Extraction Using Optimized Gabor Wavelet Based on Multi Objective Genetic Algorithm", IEEE, Department of Telecommunication Engineering, pp. 159-163, 2011.

[29] Hamed Ghodrati, Mohammad Javad Dehghani, Habibolah Danyali, "Two Approaches Based on Genetic Algorithm to Generate Short Iris Codes" ,I.J. Intelligent Systems and Applications , pp.62-79,2012.

[30] Fernando Gaxiola, Patricia Melin, Fevrier Valdez, and Oscar Castillo, "Modular Neural Networks with Type-2 Fuzzy Integration for Pattern Recognition of Iris Biometric Measure”, Springer, pp. 363-373, 2011

[31] Chung-Chih Tsai, Heng-Yi Lin, Jinshiuh Taur, and Chin-Wang Tao, "Iris Recognition Using Possibilistic Fuzzy Matching on Local Features" IEEE Transactions On Systems, Man, And Cybernetics, Vol. 42, No. 1, pp.150-162,2012.

[32] Mr. Logannathan.B, Dr. Marimuthu.A, "Iris Authentication Using PSO" International Journal of Computer\& Organization Trends, Vol. 2, pp. 10-15, 2012.

[33] Jin Liu, Xiao Fu, Xingbin Yao, "PSO-RBFNN Based Optimized PNN Classifier Model “, IEEE transaction Computer Science and Network Technology, pp. 4564592012.

[34] U. Park, R. Jillala, A. Ross, and A. K. Jain, "Periocular biometrics in visible spectrum," IEEE Trans on Information Forensic and Security, vol. 6, no. 1, pp. 96-106, 2011.

[35] V. Srivastava, B. K. Tripathi, and V. K. Pathak, “An evolutionary fuzzy clustering with minkowski distances," in Proc. Intl. Conf. on Neural Information Processing (ICONIP), 2011, pp. 753-760.

[36] G. Santos and H. Proenca, "Periocular biometrics: An emerging technology for unconstrained scenarios,"in Proc. IEEE Workshopon Computational Intelligence in Biometrics and Identity Management (CIBIM), April 2013, pp. 14-21.

[37] A. F. Sequeira, J. ao C. Monteiro, A. Rebelo, and H. P. Oliveira, "Mobbio: a multimodal database captured with a portable handheld device," Proc International Conference on Computer Vision Theory and Applications, VISAPP, vol. 3, pp. 133-139, 2014 